Vol. 5, Issue 6, June 2017

\title{
A Survey on Millimeter Wave Mobile Communications for 5G Cellular Networks
}

\author{
Deepika D Pai \\ Assistant Professor, Dept. of ECE., Vemana Institute of Technology, Bangalore, India
}

\begin{abstract}
The Present era has experienced an exponential growth in wireless mobile communication. This requires a huge bandwidth, which has motivated the utilization of millimeter wave (mm-wave) frequency spectrum to be deployed for future broadband cellular networks. Fifth generation (5G) cellular networks are being developed to satisfy the increasing data traffic among mobile devices. 5G cellular networks are expected to have much higher network capacity and provide multi-gigabits-per-second data rate for each user to support multimedia applications with stringent quality of service $(\mathrm{QoS})$ requirements. Millimeter-wave communication is a promising technology for future 5G cellular networks to provide very high data rate (multi-gigabits-per second) for mobile devices. Enabling Device to Device communications over directional $\mathrm{mm}$ wave networks is of critical importance to efficiently use the large bandwidth to increase network capacity. Basically in the traditional wireless cellular networks devices were communicating with each other through base stations, whereas the fundamental network architecture of 5G networks has the hybrid combination of $4 \mathrm{G}$ and $\mathrm{mm}$ wave communication.
\end{abstract}

Keywords: 5G; Mm Wave; High Speed Data; Propagation; Technical Challenges; 4G.

\section{INTRODUCTION}

The rapid increase in the use of smart phones and mobile data has created a huge challenge for the wireless service providers to overcome the shortage of global bandwidth. Presently the cellular providers are limited to a carrier frequency spectrum ranging between $700 \mathrm{MHz}$ and $2.6 \mathrm{GHz}$. The maximum spectrum bandwidth allocated globally for all the cellular technologies is limited to $780 \mathrm{MHz}$, where each major wireless provider has approximately $200 \mathrm{MHz}$ across all of the different cellular bands of spectrum available to them. In the modern world, to serve and interconnect users with old inefficient cell phones and the users with newer smart phones requires simultaneous management of multiple technologies in the same band-limited spectrum. At present the spectrum that is allotted for the operators is dissected into disjoint frequency bands where each band has different radio networks like third generation (3G),fourth generation(4G)and Long Term Evolution - Advanced (LTE-A) which have different propagation characteristics and building penetration losses. The challenges of future wireless communication involves:

1. Massive growth in the number of connected devices. (approximately 50 billion)

2. Massive growth in traffic volume (1000x in 10 years) due to new users / communicating machines.

3. Increasingly wide range of applications with varying requirements and characteristics: Multi-Gbps in specific scenarios, Tens of MBps almost everywhere, communicating machines.

Therefore the next generation of networks demand a need for high speed connectivity and moving to a future state of "everything everywhere and always connected".

Data services are generally discontinuous in nature. Millimeter Wave Mobile Communication for 5G Cellular allows more users to share the same resource even though the overhead associated with directing the data becomes more complex. The fixed-line network infrastructures have progressed from the use of copper wires to the virtually-limitless capacity of fiber, hence the overhead for delivering the packets in these networks are not an issue. The advances in mobile network technology and system specifications have provided higher cell capacity and consequent improvements in single user data rate. The increase in data rate will mean a consequent increase in computing power and modulation density, but since the wireless spectrum is limited, only those connections which are needed to be mobile should be wireless.

In the mobile world, capacity gains come essentially from three variables: more spectrum, better efficiency and better frequency re-use through progressively smaller cell size. It is expected that mobile data consumption will almost double year-on- year for the next five years, the network operators maintain they will struggle to meet long-term demand without even more spectrum. Freeing up frequency bands currently used for other systems will become a major priority. Mobile broadband networks need to support ever-growing consumer data rate demands and will need to tackle the exponential increase in the predicted traffic volumes. 
Vol. 5, Issue 6, June 2017

An efficient radio access technology combined with more spectrum availability is essential to achieve the ongoing demands faced by wireless carriers. In this paper, how millimeter wave can be used for $5 \mathrm{G}$ cellular is presented. The reason why the wireless community should start looking at the 3-300 $\mathrm{GHz}$ spectrum for mobile broadband applications is discussed. The propagation and device technology challenges associated with this band as well as its unique advantages for mobile communication. Introduction to millimeter-wave mobile broadband (MMB) system as a candidate for next generation mobile communication system and the feasibility for MMB to achieve gigabit-per-second data rates at a distance up to $1 \mathrm{~km}$ in an urban mobile environment is also discussed.

\section{EVOLUTION OF WIRELESS TECHNOLOGIES}

Table 2.1 briefly describes the evolution of the wireless technologies

\begin{tabular}{|c|c|c|c|c|}
\hline Name & \begin{tabular}{|l} 
1st Generation \\
Mobile Network
\end{tabular} & $\begin{array}{l}\text { 2nd Generation } \\
\text { Mobile Network }\end{array}$ & $\begin{array}{l}\text { 3rd Generation } \\
\text { Mobile Network }\end{array}$ & $\begin{array}{l}\text { 4th Generation } \\
\text { Mobile Network }\end{array}$ \\
\hline Introduced in year & $1980 \mathrm{~s}$ & 1993 & 2001 & 2009 \\
\hline Technology & $\begin{array}{l}\text { AMPS (Advanced } \\
\text { Mobile Phone } \\
\text { System), NMT, } \\
\text { TACS } \\
\end{array}$ & IS-95, GSM & $\begin{array}{l}\text { IMT2000, } \\
\text { WCDMA }\end{array}$ & LTE, WiMAX \\
\hline $\begin{array}{l}\text { Multiple Address } \\
\text { /Access system }\end{array}$ & FDMA & TDMA, CDMA & CDMA & CDMA \\
\hline Speed (data rates) & $\begin{array}{l}2.4 \mathrm{Kbps} \text { to } \\
14.4 \mathrm{kbps}\end{array}$ & $14.4 \mathrm{Kbps}$ & $3.1 \mathrm{Mbps}$ & $100 \mathrm{Mbps}$ \\
\hline $\begin{array}{l}\text { Special } \\
\text { Characteristic }\end{array}$ & $\begin{array}{l}\text { First wireless } \\
\text { communication }\end{array}$ & $\begin{array}{l}\text { Digital version of } \\
1 G \text { technology }\end{array}$ & $\begin{array}{l}\text { Digital broadband, } \\
\text { speed increments }\end{array}$ & $\begin{array}{l}\text { Very high speeds, } \\
\text { All IP }\end{array}$ \\
\hline Features & Voice only & $\begin{array}{l}\text { Multiple users on } \\
\text { single channel }\end{array}$ & $\begin{array}{l}\text { Multimedia } \\
\text { features, Video Call }\end{array}$ & $\begin{array}{l}\text { High Speed, real } \\
\text { time streaming }\end{array}$ \\
\hline Bandwidth & Analog & $25 \mathrm{MHz}$ & $25 \mathrm{MHz}$ & $100 \mathrm{MHz}$ \\
\hline
\end{tabular}

Table 2.1 Evolution of Wireless Technologies

To date, four generations of cellular communication systems have been adopted worldwide with each new mobile generation emerging every 10 years or so since around 1980: First generation analog FM cellular systems in 1981; second generation digital technology in 1993, 3G in 2001, and 4G LTE-A in 2011.

\section{Review of Previous Fourth Generations Systems:-}

A. First-Generation Systems (1G): The 1st generation was pioneered for voice service in early 1980's, where almost all of them were analog systems using the frequency modulation technique for radio transmission using frequency division multiple access (FDMA) with channel capacity of $30 \mathrm{KHz}$ and frequency band was 824-894 MHz, which was based on a technology known as Advance Mobile Phone Service (AMPS).

B. Second Generation Systems (2G): The 2nd generation was accomplished in later 1990's. The 2G mobile communication system is a digital system; this generation mainly used for voice communication also offered additional services such as SMS and e-mail. In this generation two digital modulation schemes are used; one is time division multiple access (TDMA) and the 2nd is code division multiple access (CDMA) and frequency band is 850-1900 MHz's. In 2G, GSM technology uses eight channels per carrier with a gross data rate of 22.8 kbps (a net rate of 13 $\mathrm{kbps})$ in the full rate channel and a frame of 4.6 milliseconds (ms) duration . The family of this generation includes of $2 \mathrm{G}, 2.5 \mathrm{G}$ and $2.75 \mathrm{G}$.

C. Third Generation Systems (3G): Third generation (3G) services combine high speed mobile access with Internet Protocol (IP)-based services. The main features of 3G technology include wireless web base access, multimedia services, email, and video conferencing. The 3G W-CDMA air interface standard had been designed for always-on packet-based wireless service, so that computer, entertainment devices and telephones may all share the same wireless network and be connected internet anytime, anywhere. $3 \mathrm{G}$ systems offer high data rates up to $2 \mathrm{Mbps}$, over $5 \mathrm{MHz}$ channel carrier width, depending on mobility/velocity, and high spectrum efficiency. 
D. Fourth Generation Systems (4G): $4 \mathrm{G}$ usually refers to the successor of the $3 \mathrm{G}$ and $2 \mathrm{G}$ standards. In fact, the $3 \mathrm{GPP}$ is recently standardizing LTE Advanced as future 4G standard. A 4G system may upgrade existing communication networks and is expected to provide a comprehensive and secure IP based solution where facilities such as voice, streamed multimedia and data will be provided to users on an "Anytime, Anywhere" basis and at much higher data rates compared to previous generations. One common characteristic of the new services to be provided by $4 \mathrm{G}$ is their demanding requirements in terms of QOS. Applications such as wireless broadband access, Multimedia Messaging Service (MMS), video chat, mobile TV, HDTV content and Digital Video Broadcasting (DVB) are being developed to use a $4 \mathrm{G}$ network.

E. 4G-LTE advanced: LTE also referred to as LTE-Advanced, is claimed to be the true 4G evolution step. LTE is an orthogonal frequency-division multiplexing (OFDM)-based radio access technology that supports a scalable transmission band width up to $20 \mathrm{MHz}$ and advanced multi-antenna transmission. As a key technology in supporting high data rates in 4G systems, Multiple-Input Multiple-Output (MIMO) enables multi-stream transmission for high spectrum efficiency, improved link quality, and adaptation of radiation patterns for signal gain and interference mitigation via adaptive beam forming using antenna arrays. The coalescence of HSPA and LTE will increase the peak mobile data rates of the two systems, with data rates exceeding $100 \mathrm{Mbps}$, and will also allow for optimal dynamic load balancing between the two technologies.

F. The main requirement specification for LTE advanced as approved are:

- Peak Downlink data rate: 1 Gbps, Peak Uplink data rate: 500 Mbps.

- Transmission bandwidth: Wider than approximately $70 \mathrm{MHz}$ in DL and $404 \mathrm{MHz}$ in UL.

- User throughput at cell edge 2 times higher than that in LTE.

G. Specification for Millimeter Wave Mobile Communication for 5G Cellular:

- Average user throughput is 3 times higher than that in LTE.

- Spectrum efficiency 3 times higher than that in LTE; Peak spectrum

- Efficiency downlink: 30 bps/Hz, Uplink: 15 bps/Hz

- Mobility: Same as that in LTE.

- Coverage should be optimized or deployment in local areas/micro cell

- Environments with Inter Site Distance (ISD) up to $1 \mathrm{~km}$.

\section{FIFTH GENERATION (5G) WIRELESS COMMUNICATION}

This section briefly enlists the features and advantages of the 5G Network.5G Technology stands for 5th Generation Mobile technology which has changed the means to use cell phones within very high bandwidth. It is a packet switched wireless system with wide area coverage and high throughput, using CDMA and millimeter wireless that enables a speed greater than 100Mbps at full mobility and higher than 1 Gbps at low mobility. The backbone networks of $5 \mathrm{G}$ will progress from copper and optic fiber to mm-wave wireless connections, allowing rapid deployment and mesh-like connectivity with cooperation between base stations GSMHistory.com has recorded three very distinct 5G network visions having emerged by 2014 :

1. A super-efficient mobile network that delivers a better performing network for lower investment cost.

2. A super-fast mobile network comprising the next generation of small cells densely clustered together to give a contiguous coverage over at least urban areas and gets the world to the final frontier for true wide area mobility

3. A converged fiber-wireless network that uses, for the first time for wireless Internet access, the millimeter wave bands $(20-60 \mathrm{GHz})$

A. The main features of $5 \mathrm{G}$ communication are:

- It has 10 times more capacity than others.

- Expected speed up to 1 Gbps.

- Faster and reliable when compared to 4G.

- Lower cost than previous generations.

- It offers bidirectional huge bandwidth, higher data rates and the finest Quality of Service (QOS).

B. Properties of $5 \mathrm{G}$ Networks are:

1. Worldwide cellular phone: Phone calls in any country can be done like a local call

2. Extra Ordinary data capabilities: Data capabilities of 5G systems are very much greater than existing generations and hence more data can be processed. 
UGC Approved Journal

IJIREEICE

Vol. 5, Issue 6, June 2017

3. High connectivity: The connectivity of $5 \mathrm{G}$ systems may range up to $40 \mathrm{Gbps}$

4. Greater flexibility: The mobile phones standardized in $5 \mathrm{G}$ will have the same data handling flexibility as wired systems

5. Greater amount of clarity will be present the in audio transferred in $5 \mathrm{G}$ wireless standard

6. Uses MC CDMA (Multi Carrier Code Division Multiple Access)

7. Use of Super core

8. HAPS: 5G systems will support HAPS (High Altitude Platform Stations) where UAVs (Unmanned Air vehicles) will act as intermediate transceiver stations and thus this implementation will increase the effective range of communication

9. Use of IPV6: Instead of the traditional IPV4 addressing, the new IP mode which is the IPV6 addressing will be used which will reduce the chances of having the condition where address exhaustion can occur.

10. Use of Millimeter wavebands: The portion of the electromagnetic spectrum between the IR and the microwave will be used for data propagation.

C. Architecture of 5G Network:

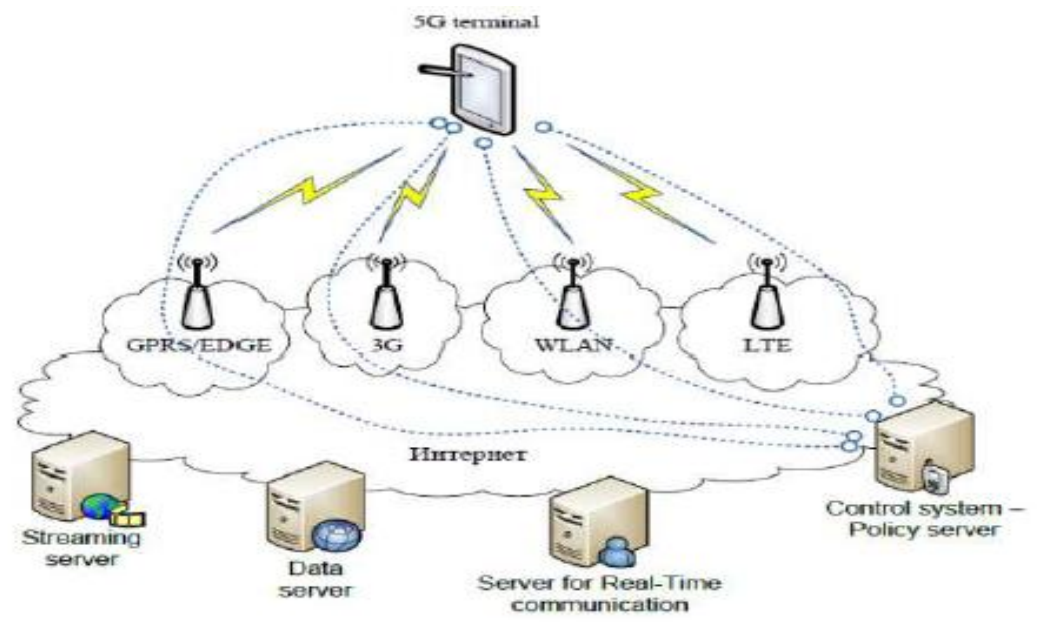

Fig 3.1 Architecture of 5G Network

Fig 3.1 gives an overview of the architecture of the 5G Network. The system comprises of a main user terminal and then a number of independent and autonomous radio access technologies. Each of the radio technologies are considered as the IP link for the outside internet world. The IP technology is designed exclusively to ensure sufficient control data for appropriate routing of IP packets related to a certain application connections i.e. sessions between client applications and servers somewhere on the Internet. The functionalities of the various clouds are as follows: GPRS (General Packet Radio Service):

a) It is mainly used to transmit data at $60 \mathrm{~kb} / \mathrm{sec}$.

b) It consumes very little battery power when sending or receiving mails or browsing the internet.

EDGE (Exchanged Data Rate for GSM Evolution):

a) It is an advanced version of the GPRS

b) It provides a data speed of $473 \mathrm{~kb} / \mathrm{sec}$.

3G (Third Generation):

a) Supports the feature of a video call on a mobile network.

b) It provides an efficient way to browse the internet using mobile networks.

WLAN (Wireless LAN):

a) It provides a short range, high speed wireless data connection between mobile data device using radio or signal.

LTE (Long Term Evolution)

a) It is the standard for mobile communication for high speed data transmission for mobile networks. It supports a speed of $100 \mathrm{Mb} / \mathrm{sec}$.

\section{G Protocol Stack:}

The figure 3.2 depicts $5 \mathrm{G}$ protocol stack showing how the $5 \mathrm{G}$ protocol layers mapped with OSI stack. As shown $5 \mathrm{G}$ protocol stack consists of OWA layer, network layer, Open transport layer and application layer. 
UGC Approved Journal

IJIREEICE

ISO 3297:2007 Certified

Vol. 5, Issue 6, June 2017

\begin{tabular}{|l|}
\hline APPLICATION LAYER \\
\hline PRESENTATION LAYER \\
\hline SESSION LAYER \\
\hline TRANSPORT LAYER \\
\hline NETWORK LAYER \\
\hline DATA LINK LAYER \\
\hline PHYSICAL LAYER \\
\hline
\end{tabular}

OSI stack

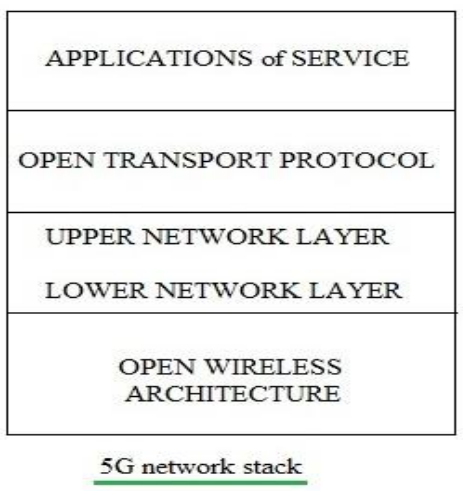

$5 \mathrm{G}$ network stack

Fig3. 2 5G Protocol Stack

OWA Layer: OWA layer is the short form of Open Wireless Architecture layer. It functions as the physical layer and data link layer of OSI stack.

Network Layer: It is used to route data from source IP device to the destination IP device/system. It is divided into lower network layer (for each interface) and upper network layers (for the mobile terminal). Here all the mobile networks will use mobile IP, with each mobile terminal acting like a foreign agent. A mobile can be attached to several mobiles or wireless networks at the same time.

Open Transport Layer: It combines functionality of both transport layer and session layer. The 5G mobile terminals have a transport layer that is possible to be down loaded and installed. Hence the name Open Transport Protocol.

Application Layer: It marks the data as per proper format required. It also does encryption and decryption of the data. It selects the best wireless connection for given service.

\section{Hardware of 5G:}

a) Uses Ultra Wide Band networks with higher Bandwidth (4000 Mbps) at low energy levels.

b) Uses smart antennas.

\section{Software of 5G:}

a) Wireless networks including LAN technologies, LAN/WAN, World Wide Wireless Web, unified IP and Software defined radio.

b) Encryption, flexibility, Anti-virus.

\section{AN INTRODUCTION TO MILLIMETER (MM) WAVE TECHNOLOGY}

\section{Millimeter wave spectrum for 5G}

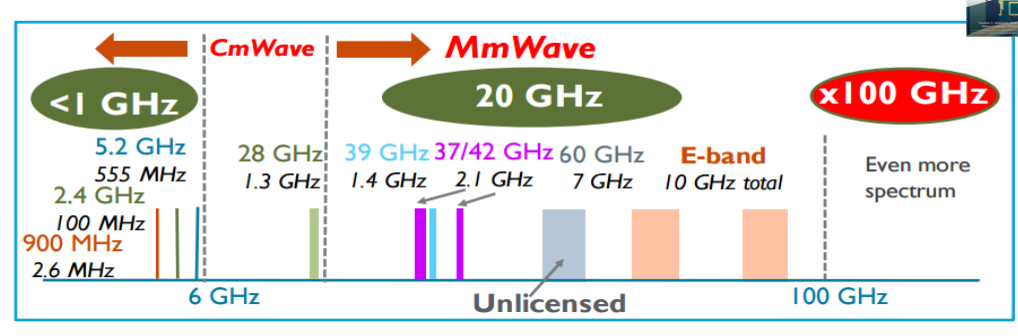

Fig 4.1 Millimeter Wave Spectrum.

Mm Wave is a promising technology for future cellular systems. The frequency bands which lies between $30 \mathrm{GHz}$ to $300 \mathrm{GHz}$ is known as millimeter wave as the wavelength of electro-magnetic wave will be in millimeter range at these frequencies. As there has been a rapid increase in the number of mobile data subscribers, a need for larger bandwidth also arises. Presently rhe available mobile frequency spectrum which is below the $\mathrm{mm}$ band has a limited bandwidth. Due to this millimeter wave band has been explored by the mobile operators as mobile frequency spectrum as it supports larger bandwidth. Though penetration loss is higher at these $\mathrm{mm}$ wave frequencies as these frequencies cannot penetrate walls and certain objects in the buildings. Moreover $\mathrm{mm}$ wave frequencies get attenuated due to rain.

Since there is a limited spectrum availability for commercial cellular systems, most researches are focused on increasing spectral efficiency by using OFDM, MIMO, efficient channel coding, and interference coordination. Three key characteristics distinguish millimeter wave communication from sub-6 $\mathrm{GHz}$ communication: (1) the specular 
UGC Approved Journal

IJIREEICE

Vol. 5, Issue 6, June 2017

nature of propagation, (2) the resultant need for large multi-antenna arrays at both ends of the link, and (3) the availability of ultra-wide bandwidth channels. The millimeter wave frequencies which are used for 5G mobile technology is known as $5 \mathrm{G}$ millimeter wave.

A. $\quad 5 \mathrm{G}$ millimeter wave technology features

Table 4.1 Features of 5g Millimeter Wave Technology.

\begin{tabular}{|l|l|}
\hline Features & Description \\
\hline Data rate & $10 \mathrm{Gbps}$ or higher \\
\hline Frequency Bands & The bands are split into $<40 \mathrm{GHz}$ and $>40 \mathrm{GHz}$ up to $100 \mathrm{GHz}$ frequency. \\
\hline Bandwidths & $\begin{array}{l}\bullet 10 \mathrm{subcarriers} \text { of } 100 \mathrm{MHz} \text { each can provide } 1 \mathrm{GHz} \mathrm{BW} \text { due to carrier aggregation at } \\
<40 \mathrm{GHz} \\
\cdot 500 \mathrm{MHz} \text { to } 2 \mathrm{GHz} \mathrm{BW} \text { can be achieved without carrier aggregation at }>40 \mathrm{GHz} .\end{array}$ \\
\hline Distance coverage & 2 meters (indoor) to 300 meters (outdoor) \\
\hline Modulation types & CP-OFDMA<40GHz SC $>40 \mathrm{GHz}$ \\
\hline Frame topology & TDD \\
\hline latency & About $1 \mathrm{~ms}$. \\
\hline MIMO type & $\begin{array}{l}\text { Massive MIMO is supported. Antennas are physically small and hence there will be } \\
\text { approx. } 16 \text { antenna array available in 1 square inch. Hence } 5 \mathrm{G} \text { mm wave compliant } \\
\text { eNBs support } 128 \text { to } 1000 \text { antenna arrays. These are used to increase both the capacity } \\
\text { and coverage. }\end{array}$ \\
\hline
\end{tabular}

B. $\quad 5 \mathrm{G}$ millimeter wave frame structure

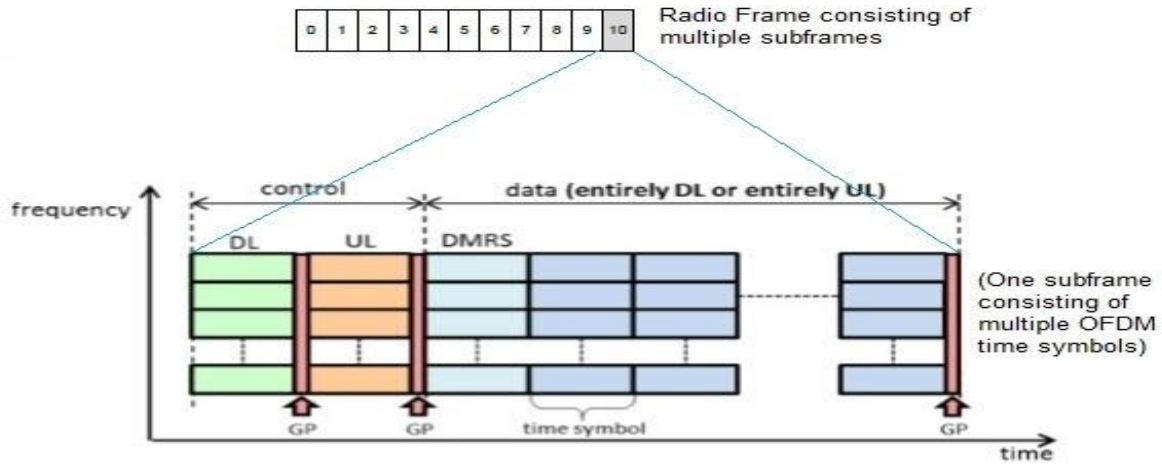

Fig4.2 5G millimeter Wave Frame and Subframe

Fig 4.2 explains the frame structure of $5 \mathrm{G}$ millimeter wave.5G millimeter wave frame follows TDD topology. The TDD refers to Time Division Duplex. The frame duration is about 10ms. The frame is composed into smaller size sub frames (of duration 1ms). The sub frame is made up of OFDMA symbols. Here both downlink (i.e. transmissions from eNB to UEs) and uplink (i.e. transmissions from UEs to eNB) are allocated different time instances in the sub frame. Both downlink and uplink uses same RF frequencies in TDD topology at different time slots. There will be GP (Guard Period or Gap) between different parts of sub frame to avoid interference. The sub frame is divided into reference, control and data parts. Reference signals are carried in each sub frame are used to demodulate downlink and uplink data parts. Control part carries messages used for connection establishment and management between eNB and UE. Data part carries data payload as per application of usage by various UEs in $5 \mathrm{G}$ system. Sub frame is further divided based on downlink only (DL only), uplink only (UL only) and bi-directional usage by UEs and eNB.

\section{Advantages of $5 \mathrm{G}$ millimeter wave:}

Following are the advantages/merits of the $5 \mathrm{G}$ millimeter wave. These benefits make $5 \mathrm{G}$ in millimeter wave as one of the strong contender for the future of mobile wireless communication domain.

- Provides larger bandwidth and hence more number of subscribers can be accommodated.

- Due to less bandwidth in millimeter range, it is more favorable for smaller cell deployment.

- Coverage is not limited to line of sight as first order scatter paths are viable.

- Channel sounding feature is employed to take care of different types of losses at mm wave frequencies so that $5 \mathrm{G}$ network works satisfactorily. Channel sounding refers to measurement or estimation of channel characteristics which 


\section{International Journal of Innovative Research in Electrical, Electronics, Instrumentation and Control Engineering}

ISO 3297:2007 Certified

Vol. 5, Issue 6, June 2017

helps in successful design, development and deployment of $5 \mathrm{G}$ network with necessary quality requirements. - Antenna size is physically small and hence large number of antennas are packed in small size. This leads to use of massive MIMO in eNB/AP to enhance the capacity.

- Dynamic beam forming is employed and hence it mitigates higher path loss at $\mathrm{mm}$ wave frequencies.

- 5G millimeter wave networks support multi-gigabit backhaul up to 400 meters and cellular access up to 200-300 meters.

Due to these benefits, $5 \mathrm{G} \mathrm{mm}$ wave is suitable for mobile communication over sub-6GHz wireless technologies.

D. Disadvantages of $5 \mathrm{G}$ mm wave:

Following are the disadvantages/demerits of the $5 \mathrm{G}$ millimeter wave.

- Millimeter wave goes through different losses such as penetration, rain attenuation etc. This limits distance coverage requirement of $\mathrm{mm}$ wave in $5 \mathrm{G}$ based cellular mobile deployment. Moreover path loss at $\mathrm{mm}$ is proportional to square of the frequency. It supports 2 meters in indoors and about 200-300 meters in outdoors based on channel conditions and AP/eNB height above the ground.

- Supports only LOS (Line of Sight) propagation. Hence coverage is limited to LOS.

- Foliage loss is significant at $\mathrm{mm}$ wave frequencies.

- Power consumption is higher at millimeter wave due to more number of RF modules due to more number of antennas. To avoid this drawback, hybrid architecture which has fewer RF chains than number of antennas need to be used at the receiver. Moreover low power analog processing circuits are designed in $\mathrm{mm}$ wave hardware.

\section{E. SECURITY:-}

Since millimeter waves have a narrow beam width and are blocked by many solid structures they also create an inherent level of security. In order to sniff millimeter wave radiation a receiver would have to be setup very near, or in the path of, the radio connection. The loss of data integrity caused by a sniffing antenna provides a detection mechanism for networks under attack. Additional measures, such as cryptographic algorithms can be used that allow a network to be fully protected against attack.

\section{CONCLUSION AND FUTURE SCOPE}

An overview of using Millimeter wave Mobile Communication for 5G Cellular is presented in this paper, and how 5G Cellular systems can overcome the issues related to the previous generations of Communication systems and evolved to be the most promising System. Given the worldwide need for cellular spectrum, and the relatively limited amount of research done on mm-wave mobile communications, fact that the large bandwidth available at millimeter wave frequencies results in very high data transmission rate; also helps to minimize the amount of time that a node needs to stay in transmission mode; and therefore, minimizes the possibility of its transmission being detected. The security and reliability provided is quite huge. Hence considering all the factors given above these millimeter wave frequencies is going to serve the future generations of wireless communications enabling the "ALL IP" features and providing good quality of service (QOS). $28 \mathrm{GHz}$ and $38 \mathrm{GHz}$ are the current frequencies that have low rainfall attenuation \& atmospheric attenuations. Further research must take place in this band and the characteristics of other frequencies needs to be studied, the penetration power and the range for communication needs to be further improved.

\section{REFERENCES}

[1] T. S. Rappaport, Shu Sun, Rimma Mayzus et al "Millimeter wave mobile communications for 5G cellular: it will work!," Proc. IEEE, vol. 1, 2013, no. 10, pp.335_349, may. 2013.

[2] T. S. Rappaport, J. N. Murdock, and F. Gutierrez, "State of the art in $60 \mathrm{GHz}$ integrated circuits \& systems for wireless communications," Proc.int IEEE, vol. 99, no. 8, pp. 1390_1436, Aug. 2011.

[3] M. Cudak, A. Ghosh, T. Kovarik, R. Ratasuk, T. Thomas, F. Vook, and P. Moorut, “Moving towards mmwave-based beyond-4G (B-4G) Technology," in Proc. IEEE Veh. Technol. Soc. Conf., 2013, pp. 1_17.

[4] Y. Chen, S. De, R. Kernchen, and K. Moessner, "Device discovery in future service platforms through SIP," in Proc. IEEE Veh. Technol. Conf., Sep. 2012, pp. 1_5. URL's:

[5]http://www.cablinginstall.com/articles/2013/12/millimeter-wave-article.html

[6]http://nsn.com/news-events/insight-newsletter/articles/5g-ultra-wideband-enhanced-local- 24 area-systems-at-millimeter-wave

[7]http://global.samsungtomorrow.com/?p=24093

8] http://www.mobileinfo.com/3G/4G_Sun_MobileIP.htm

[9] http://www.athenawave.com/products/about-millimeter-wave

[10] http://www.profheath.org/hot-topics/millimeter-wave-cellular-systems

[11] Engineering and Technology History Wiki, (2015, Sep.17), Millimeter Waves, Available: http://www.ethw.org/Millimeter Waves

[12] Equinox telecom, (N.D), MILLI-METER WAVE / EBAND 5G WIRELESS SOLUTIONS. 